\title{
Uso de redes sociales como elemento de interacción y construcción de contenidos en el aula: cultura participativa a través de Facebook
}

\author{
Erika P. Alvarez-Flores \\ Universidad Estatal de Sonora (México) \\ ericka.alvarez@ues.mx \\ Patricia NúÑEZ GóMEZ \\ Universidad Complutense de Madrid (España) \\ pnunezgo@ucm.es
}

\section{Resumen}

De cara al S.XXI, es necesario innovar dentro de las aulas con recursos utilizados por los nativos digitales. La innovación debe presentarse como elemento motivador, por lo que implementarla es un gran reto. Las redes sociales han permitido que estudiantes y docentes interactúen de una manera más natural al usar lenguajes que utilizan para actividades fuera del aula. En esta Investigación se pretende conocer si la estrategia de implementación de Facebook en las clases, es aceptada por los estudiantes como una herramienta más para el proceso de enseñanza-aprendizaje en el ámbito universitario.

Palabras clave: Innovación educativa, tecnologías, redes sociales, Facebook.

Social Networks as an element of interaction and content building in the classroom: Participatory culture through Facebook.

\begin{abstract}
:
Today, it is necessary to innovate in the classroom with resources used by digital natives. This innovation must be present as a motivator in classrooms; therefore, to implement it is currently a challenge. The social networks have allowed to the teachers interact with students in a more natural way, using the same language that they use for their activities outside the classroom. In this research, we expect to know if students accept the strategy of Facebook implementation in the classroom, as a tool for teaching-learning process in the university.
\end{abstract}

Key Words: Educational innovation, technology, social networks, Facebook.

\section{Referencia normalizada:}

Alvarez Flores, E. P.; Núñez Gómez, P. (2013) Uso de redes sociales como elemento de interacción y construcción de contenidos en el aula: cultura participativa a través de Facebook. Historia y Comunicación Social. Vol. 18 Nº Especial Noviembre. Págs. 53-62

Sumario: 1. Introducción. 2. Web Social o Web 2.0. en la educación 3. Metodología. 3.1. Grupo de estudio. 3.2. Definición de procedimientos de medición. 4. Los estudiantes universitarios y Facebook. 5. Conclusiones. 6. Referencias bibliográficas. 7. Anexo. 


\section{Introducción}

La revolución de las tecnologías de la comunicación ha permitido la incorporación de una diversidad de estrategias y mecanismos pedagógicos en el proceso de enseñanza y aprendizaje. Aún que la innovación educativa no lleva el mismo ritmo de la innovación tecnológica, los retos que imponen las nuevas tecnologías de la Información y de la Comunicación (TICs) han sido enfrentados progresivamente por millones de usuarios, de ahí que las oportunidades que ofrecen las redes sociales o Web 2.0 han sido aprovechadas al máximo por algunos estudiantes y especialmente por profesores.

Las características que presentan sitios en la red como Facebook, MySpace y Twitter, así como su popularidad entre los jóvenes y el tiempo empleado por ellos, han propiciado que las instituciones educativas estén considerando esta nueva estructura social como una más de las herramientas en la docencia, con el objetivo de facilitar el proceso de aprendizaje. La utilización correcta de esta tecnología ofrece canales formativos distintos a los empleados tradicionalmente. Si bien este tipo de sitios en la red no fueron concebidos específicamente para uso académico, están siendo identificados como una tecnología emergente para enseñar y aprender. Investigaciones en este ámbito han demostrado como las redes sociales a través de su amplia variedad de elementos estén permitiendo que se lleven a cabo actividades educativas y que los estudiantes al colaborar y comunicarse más con el profesor y entre ellos mismos, sean protagonistas de su propio aprendizaje. Haciendo posible además entre otras cosas, desarrollar en los estudiantes un pensamiento crítico, como así afirman Ajjan y Hartshorne (2008); Griffith y Liyanage (2008). En este mismo sentido, Munoz y Towner (2009) aseguran que redes como Facebook generan un impacto positivo en la vida de los estudiantes al utilizarlo para compartir información concerniente a las asignaturas de su trayectoria académica así como para proyectos independientes de grupo.

No obstante la popularidad de estas tecnologías por parte de los profesores como elemento de interacción y construcción de contenidos en el aula, es de suma importancia considerar además el asentimiento por parte de los estudiantes. Indudablemente, el conocer el grado de aceptación que tienen los jóvenes en relación a las redes sociales (como Facebook) en el desarrollo de actividades educativas, permitirá identificar claramente que estrategias propiciarán una adecuada práctica académica al integrarlas dentro de las asignaturas en el ámbito de educación superior.

\section{Web social o web 2.0 en la educación}

En la actualidad, personas altamente heterogéneas, de diferente género, edad, nivel de educación, idioma, cultura y sobre todo individuos jóvenes, han incorporado a su vida cotidiana las redes sociales. Investigaciones sobre el uso y las actitudes hacia Internet, indican que estudiantes universitarios son los usuarios más asiduos de Internet, específicamente de sitios web como Facebook, MySpace y de motores de 
búsqueda, Rhoades et al (2008). En este mismo sentido, Hoover (2008) manifiesta el cómo estudiantes de primer año en un instituto de enseñanza superior acostumbran utilizar Facebook; mientras que en un análisis de contenido cuantitativo realizado por Kolek y Saunders (2008), también se identifican una amplia mayoría de cuentas en Facebook por parte de estudiantes universitarios. La rápida adopción de estos medios sociales principalmente entre los jóvenes ha propiciado por ende que los profesores estén interesados en utilizarlas con propósitos educativos, específicamente en las universidades con el fin de mejorar la participación de los alumnos.

El término "Web 2.0" o "Web Social" fue instaurado en 2004 por Tim O'Reilly, en referencia a una segunda generación de Web basada en comunidades de usuarios dentro de una gama especial de servicios, como blogs o los wikis y las mencionadas redes sociales, donde todos ellos promueven una alta colaboración e intercambio de información entre usuarios. De ahí, que la estructura de la transferencia de información a través de las redes sociales ha abierto la puerta a una nueva distribución del poder.

La incorporación de estudiantes a redes donde puedan compartir su conocimiento y experiencias relacionadas con un campo en particular, se enriquece continuamente con las contribuciones que realicen cada uno de ellos. Con todo, la oportunidad que brindan las redes sociales para una educación de calidad es conectar y construir una fuerte relación entre la alfabetización digital que los estudiantes ya poseen y combinarla con la clase tradicional, como afirma Edmondson (2012). Según Hew (2011), existen esencialmente nueve elementos que motivan a los estudiantes para recurrir a las redes sociales: por diversión, para tratar de ser uno de los más populares, para mantener contacto con gente que no ven con frecuencia, conocer gente nueva, aprovechar el tiempo libre, incursionar en la sociedad y con fines de enseñanza. Principalmente, como herramienta para gestionar fotos, sus contactos y otra información personal. Inclusive hasta para actividades electorales. Sin embargo, de los nueve motivos, los jóvenes utilizan Facebook especialmente para las relaciones sociales, Mazman y Usluel (2011).

Diversos estudios basados en el uso de redes sociales han surgido con el fin de ayudar a los educadores a interactuar mejor con los estudiantes en Facebook. En este sentido, un estudio del 2009 ha explorado el potencial de estos sistemas en cursos universitarios, encontrando Ophus y Abbitt que la utilización de este tipo de sistemas es altamente viable. Mazman y Usluel (2010) describen que el factor más relevante para elegir la adopción de Facebook en la educación está estrechamente relacionado con su utilidad, además de considerar las habilidades que tienen los jóvenes para utilizarlo, así como su influencia social, facilitando con ello las condiciones e identidad de sus usuarios. Según Aydin (2012), Facebook es una red colaborativa para comunicarse, reclutar, compartir conocimientos, y sobre todo para mantener las relaciones existentes. Por su parte, Mazman y Usluel (2011) lo consideran viable para crear nuevas relaciones, ayudarse con fines académicos y dar seguimiento a programas específicos. Inclusive, Facebook incrementa el compromiso del estudiante a través del desarrollo de comunidad en el aula y estimulando el discurso intelectual, Hurt et al 
(2012). Las redes sociales también generan diversas oportunidades que incrementan la motivación a los estudiantes involucrándolos en el proceso de aprendizaje, Siegle (2011). En definitiva, los estudiantes pueden sentirse más cómodos usando Facebook para expresar sus opiniones y simplemente, los profesores tienen que ayudarles a mejorar la participación para lograr las metas educativas, Junco (2012).

No obstante, cabe destacar lo escaso de las actividades de carácter académico realizadas por los estudiantes a través de Facebook. En la Literatura se ha encontrado además, que la capacidad de atención de los estudiantes tiene significativamente impacto en sus calificaciones. Estando altamente influenciado el resultado por la percepción que éstos tengan a cerca de las redes sociales, lo que les gusta o les disgusta de ellas, así como la facilidad de su uso, Paul et al (2012). En este sentido, algunas investigaciones han revelado acerca de posibles efectos negativos del uso de Facebook en la educación. Es decir, se piensa que en lugar de suscitar una cultura participativa académica ha influido a incrementar los elementos de distracción de los estudiantes, Wise et al (2011). Otro de ellos, describe que no es común que los estudiantes utilicen Facebook con el objetivo específico de estudiar, repercutiendo realmente en sus resultados académicos, Kirschner y Karpinski (2010).

Dado que los sitios de redes sociales son considerados como un elemento favorable de interacción y construcción de contenidos fuera y dentro de las aulas de clases, y siguiendo las nuevas tendencias pedagógicas planteadas por el Espacio Europeo de Educación Superior (EEES), un conjunto de docentes de la Universidad Complutense ha recurrido a Facebook como apoyo a sus asignaturas. En un esfuerzo por aprovechar de manera óptima los recursos que brindan estas tecnologías de información y comunicación, en este estudio se pretende poner en claro la aceptación que tiene la implementación de Facebook para actividades académicas por parte de estudiantes universitarios, específicamente conocer el grado de aprobación como elemento académico y sobre todo si la consideran como recurso viable para satisfacer sus propios intereses académicos y por ende para adquirir conocimientos.

\section{Metodología}

\subsection{Grupo de estudio}

Un total de ciento veinticuatro estudiantes de la Facultad de Ciencias de la Información de la Universidad Complutense de Madrid fueron invitados a colaborar en este estudio. Esencialmente, a estudiantes que sus profesores combinaron Facebook con la clase tradicional, como una herramienta de apoyo en alguna de sus asignaturas. De los 124 participantes del grupo de estudio, 81 corresponden al primer curso de publicidad y 43 corresponden al tercer curso (estudiantes que utilizaron esta estrategia anteriormente). Debido al perfil del programa educativo, el grupo estaba conformado en su mayoría por estudiantes de género femenino (74.19\%), donde además las edades de todos los participantes oscilan entre los 18 y 20 años. Los profesores 
instituyeron la estrategia, basada en cultura participativa, en los cursos académicos 2011/2012 y 2012/2013.

\subsection{Definición de procedimientos de medición}

Se diseñaron páginas en Facebook propias de cada asignatura al inicio de los dos cursos académicos objetos de investigación. Sólo los profesores y estudiantes involucrados en cada una de las asignaturas tenían acceso al perfil privado. En éstas, se instituyeron una variedad de materiales interesantes para los estudiantes en relación a su perfil académico y de temas relacionados con la asignatura, con el fin de realizar una serie de actividades académicas.

La estrategia para la recolección de datos consiste en un cuestionario en papel aplicado a los estudiantes que forman el grupo de estudio, enfocándose esencialmente a medir aspectos que manifiesten el grado de aceptación y satisfacción adquirida derivada de la experiencia de las redes sociales en el ámbito académico. A través de éste, se busca esencialmente que se aporte evidencia para implementar nuevas estrategias para el desarrollo de actividades en clases.

Por lo que, el cuestionario ha sido dividido en tres secciones: En el primer segmento, se especifican preguntas básicas relacionadas con la frecuencia del uso de las redes sociales en cuestiones académicas así como el tipo de usuario que se considera el mismo estudiante. En la segunda parte se utilizó una escala tipo Likert de 5 puntos con el fin de revelar claramente qué tipo de factores académicos están implicados en la utilización de Facebook. Adicionalmente, el cuestionario también envuelve una sección con preguntas abiertas para que los participantes expresen libremente su opinión sobre los beneficios y/o problemas que han identificado cuando se han implementado las redes sociales en sus cursos a nivel universitario. La aplicación del cuestionario tuvo lugar al finalizar las diversas asignaturas en junio de 2013.

\section{Los estudiantes universitarios y Facebook}

Los datos expuestos tras la investigación revelan que a pesar de que el $70.97 \%$ de los participantes aún se describen como estudiantes tradicionales, más del $76 \%$ están interesados en seguir adscritos a grupos de Facebook orientados exclusivamente a asuntos de carácter académico. Esto, con el objetivo único de tener acceso a través de él a notas y a otro tipo de materiales relacionados con sus cursos, así como mantener comunicación con sus compañeros que comparten las mismas asignaturas, generando una cultura participativa a cualquier hora y desde cualquier lugar fuera del aula de clases. Otros resultados indican que la interacción en Facebook para estos estudiantes nativos digitales que están acostumbrado a utilizarlo, les resulta sobre todo entretenida y fácil. Al no implicarles mucho esfuerzo, el 52.42\% recurrieron aproximadamente a una hora al día en Facebook para trabajar en grupos de estudio, mientras que un $33.87 \%$ utilizaron alrededor de 1 a 3 horas y sólo un $8.87 \%$ mencionaron que 
necesitaron más de 3 horas. Encontrándose no obstante, un porcentaje de $4.84 \%$ de nativos digitales a quienes no les interesó participar en ella.

A pesar de lo anterior, un bajo índice del $16.13 \%$ no están de acuerdo en que Facebook les ha facilitado la organización de estudio y tiempo libre. Una interesante observación es la diferencia de opinión entre los estudiantes de primero y los de tercero, en relación al hecho de considerar útil las redes sociales como una herramienta para un estudio eficaz. Es decir, el $41.98 \%$ de los estudiantes de primero testifican que saben obtener el mejor rendimiento de la red social para su estudio, mientras que la experiencia de los estudiantes del tercer año les permite alcanzar hasta el $62.79 \%$ en este factor. No obstante, el $73.39 \%$ de todos los participantes han aceptado que Facebook realmente si les ha ayudado en el desarrollo de cuando menos una de sus asignaturas en la universidad.

Por otra parte, analizando las opiniones sobre los beneficios y/o problemas suscitados para la construcción de contenidos a través de redes sociales, se observa claramente como un alto porcentaje de los estudiantes ven viable seguir utilizando específicamente Facebook como una herramienta educativa. Entre las principales razones especificadas por el $84.68 \%$ de los participantes se encuentra la buena comunicación que surge entre ellos mismos y con el profesor para compartir contenidos relacionados con los distintos tópicos de la asignatura. Al hacerse más ameno el método de trabajo, se ha logrado estimular al $75 \%$ de los estudiantes que ahora perciban los cursos de modo interesante mientras logran resolver sus dudas con solo tener una cuenta en la red.

En este mismo tenor, el $83.87 \%$ de todos los participantes consideran que Facebook promueve una comunicación más activa y abierta que favorece la difusión de la información, de ahí que puede ser útil para transferir conocimientos más fácilmente en sus próximos cursos. Un dato a destacar en este sentido es que el porcentaje de estudiantes de tercer año, que ya previamente emplearon la red social en otro ciclo escolar, es más bajo $(72.09 \%)$ en comparación a los estudiantes de primer año $(90.12 \%)$. A pesar de ello, el $51.16 \%$ de los alumnos de tercer año prefieren Facebook en sus cursos en lugar de otras redes académicas, registrándose el $48.15 \%$ en los de primer año. Uno de los participantes expone que "Da menos pereza entrar a Facebook que a la red virtual de la facultad".

Otras ventajas que hacen viable seguir utilizando Facebook como una herramienta educativa en las universidades son señalas en el Gráfico 1. 
Gráfico 1: Características que favorecen la aceptación del uso de Facebook en las asignaturas.

\section{VENTAJAS DE UTILIZAR FACEBOOK EN ASIGNATURAS SEGÚN ESTUDIANTES}

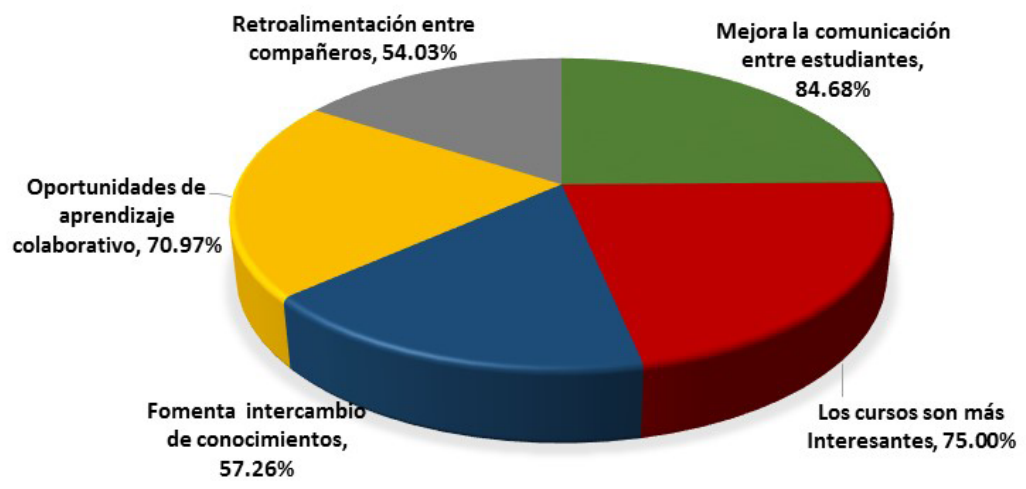

Fuente: Elaboración propia

Aunado a esto, el $66.94 \%$ de los participantes concuerdan en afirmar que las actividades didácticas desarrolladas eficazmente por los profesores proporcionan un mejor ambiente de aprendizaje. Pero aseguran que esto se llevará a cabo, siempre y cuando el profesor utilice correctamente los recursos de las redes sociales. En este sentido, los estudiantes revelan que requieren una mayor interacción o participación de los docentes en el sitio para lograr un sistema de aprendizaje que les permita alcanzar niveles de excelencia. Por ejemplo, uno de los participantes comenta que "Promoviendo un mayor control en la información a establecer en Facebook, seremos más productivos".

Aunque los resultados demuestran que los estudiantes son conscientes de las herramientas y los recursos que tiene Facebook para facilitar el proceso de enseñanza-aprendizaje, también exponen que existen elementos que están impactando negativamente y que a los estudiantes les gustaría que fuesen considerados para aceptar totalmente la integración de las redes sociales en las asignaturas.

Específicamente, para algunos estudiantes es inadecuado este tipo de herramientas ante la posibilidad de que se revelen aspectos de su vida privada a personas que no desean. Por otro lado, un $67 \%$ considera estos sitios causantes de distracciones, al identificarlos solo en plan de ocio y no de propósito serio. Uno de los estudiantes expresa "Tenemos en el mismo sitio las cosas personales y otras herramientas como juegos que hacen que acabemos distrayéndonos y dejemos a un lado las actividades académicas". Otro especifica "No me siento a gusto que los profesores se enteren de tu vida personal al agregarlos como amigos". En este caso, sugieren establecer cuentas específicas, una de propósito personal y otra alternativa para el ámbito educativo. 
De igual forma, manifiestan la presencia de alumnos que crean confusiones al hacer uso inadecuado de Facebook, por ejemplo, cuando establecen información incompleta o no del todo cierta. No obstante sean nativos digitales, también existe la posibilidad de que haya estudiantes que no estén habituados a este tipo de herramientas y se les dificulte su utilización, creándose una desventaja en relación a sus otros compañeros.

\section{Conclusiones}

Los estudiantes están conscientes del potencial que tienen estos entornos virtuales y los consideran viables para facilitar el proceso de adquisición de conocimientos y satisfacer parte de sus intereses académicos. Aunque la aceptación de la herramienta Facebook es mayoritaria por parte de los estudiantes como apoyo en el aula, sigue habiendo algún alumno que prefiere no utilizarla por motivos diversos: privacidad, desconocimiento, poco fiable.

El uso de redes sociales en el aula aún tiene varios problemas que resolver en cuanto a la implicación del alumno y su creencia de que la escuela debe seguir utilizando medios tradicionales y no debe estar vinculada al ocio. Los resultados destacan que la clave de éxito de esta innovación en el proceso enseñanza-aprendizaje es el tipo de estrategias que apliquen los profesores para motivar a los estudiantes y emocionalmente implicarlos en las actividades académicas establecidas en el sitio web. Es fundamental para ello, el grado de involucramiento que tenga el docente en las actividades y cómo utiliza éste la herramienta.

De ahí que el profesor debe planear estratégicamente los objetivos de esta red, aunarlos con los de la asignatura y motivar a través de diferentes indicadores al alumno. Si no es así, la participación y el aprendizaje no serán adecuados.

En concreto, la experiencia generada al integrar Facebook como parte del proceso educativo ha permitido canales formativos distintos a los tradicionales y han potenciado entre los estudiantes una comunicación más abierta y activa al expandir su creatividad y conocimientos. Los estudiantes si consideran viable integrar redes sociales en procesos formales de enseñanza superior si la misma universidad y los profesores promuevan estos tipos de herramientas. Es decir, que la conciban como una práctica "normal", donde más profesores de otras asignaturas participen en esta práctica.

\section{Referencias bibliográficas}

ABUIN, A. (2009). "Las redes sociales como herramienta educativa en el ámbito universitario". En: Relada, Vol.3, nº 3, p. 1-8.

AJJAN, H.; HARTSHORNE, R. (2008). "Investigating faculty decisions to adopt Web 2.0 technologies: theory and empirical tests". En: The Internet and Higher Education, vol. 11(2), p. 71-80. 
AYDIN, S (2012). "A review of research on Facebook as an educational environment”. En: Education Tech Research Dev (2012), vol. 60, p .1093-1106.

EDMONDSON, E. (2012). "Wiki Literature circles: Creating digital Learning communities". En: English Journal, vol. 101(4), p. 43-49.

HEW, K. F. (2011). "Students' and teachers' use of Facebook". En: Journal Computer in Human Behavior, vol. 27, p. 662-676.

GRIFFITH, S.; LIYANAGE, L. (2008). "An introduction to the potential of social networking sites in education". En: Emerging Technologies Conference 2008.

HOOVER, E. (2008). "Colleges face tough sell to freshmen, survey find". En: Chronicle of Higher Education, vol. 54(21).

HURT, N. E., (et al.) (2012). "The "Facebook Effect: College Students"e Perceptions of Online Discussions in the Age of Social Networking". En: International Journal for the Scholarship of Teaching and Learning, vol. 6(2), 1-24.

JUNCO, R. (2012). "The relationship between frequency of Facebook use, participation in Facebook activities and students engagement". En: Journal Computers \& education, vol. 58, p. 162-171.

KIRSCHNER, P. A.; KARPINSKI, A. C. (2010). "Facebook and academic performance". En: Journal Computers in Human Behavior, vol. 26, p. 1237-1245.

KOLEK, E. A.; SAUNDERS, D. (2008). "Online disclosure: An empirical examination of undergraduate Facebook profiles". En: NASPA Journal, no. 45(1), p. 1-25.

MAZMAN, S. G.; USLUEL, Y. K. (2010). "Modeling educational usage of Facebook". En: Journal Computer \& Education, vol. 55, p. 444-453. - (2011). "Gender differences in using social networks". En: Turkish Online Journal of Educational Technology, vol. 10(2), p. 133-139.

MUÑOZ, C.; TOWNER, T. (2009). "Opening Facebook: How to use Facebook in the college classroom". En: Proceedings of society for information technology and teacher education international conference 2009, p. 2623-2627.

OPHUS, J. D.; ABBITT, J. T. (2009). "Exploring the Potential Perceptions of Social Networking Systems in University Courses". En: Journal of Online Learning and Teaching, Vol. 5 (4), p. 639-648.

PAUL, J. A.; BAKER, H. M.; COCHRAN J. D. (2012). "Effect of online social networking on student academic performance". En: Journal Computers in Human Behavior, vol. 28, p 2117-2127.

RHOADES, E. B., (et al.) (2008). "Internet as information source: Attitudes and usage of students enrolled in a college of agriculture course". En: Journal of Agricultural Education, vol. 49(2), p. 108-117.

SIEGLE, D. (2011). "Facing Facebook: A guide for non-teens". En: Gifted Child Today, vol. 34(2), p. 14-19.

WISE L.; SKUES J.; WILLIAMS B. (2011). "Facebook in higher education remotes social but not academic engagement". En: Proceedings ascilite 2011 Hobart, p. 1332-1342. 


\section{Las autoras}

Erika P. Alvarez Flores. Profesora Titular en la Universidad Estatal de Sonora, Unidad Académica Hermosillo, México. Doctor en Tecnologías Multimedia por la Universidad de Granada, España.

Patricia Núñez Gómez. Profesora Titular de Publicidad, Facultad de Ciencias de la Información, UCM. Doctor en Publicidad y Relaciones públicas por la Universidad Complutense. Miembro de diferentes grupos de investigación www.gruposocmedia. es; www.grupofonta.org, www.grupopalabraseimagenes.uah, Directora del grupo Brandingkids. Miembro de diversas asociaciones de investigación internacionales (Ecrea, Ica,) 